\title{
Cardiac Tamponade Presenting as Abdominal Pain and Being the Initial Manifestation of Malignant Disease: A Case Report
}

\author{
Tomás Belzunegui ${ }^{\mathrm{a}}$ b, c, Elena Alvarez ${ }^{\mathrm{a}}$, Elena Baztarrica ${ }^{\mathrm{a}}$, Paula Sota ${ }^{\mathrm{a}}$
}

\begin{abstract}
Neoplastic pericarditis can be presented as acute pericarditis, pericardial effusion, effusive constrictive pericarditis or cardiac tamponade. For the majority of patients, a clinical manifestation of neoplastic pericarditis is absent or remains unrecognised during their life. A 56 year old male asymptomatic until three days ago that began with intense pain in epigastric region. The yugular vein was distended, breath and heart sounds were slightly decreased. Right-sided abdominal pain especially pronounced in the epigastric and right iliac fossa, his liver was palpable $2 \mathrm{~cm}$ below the right costal margin. In first cut of abdominal CT we showed pericardial effusion. The patient improved immediately after thetherapeutic pericardiocentesis. For the study of primary tumor was performed a thoraco-abdominal CT. Showing right hiliar mass, mediastinal lymph nodes, bilateral lung metastases. The patient was diagnosed with stage IV lung carcinoma, initiated the chemotherapy treatment (carboplatin-gemcitabine) by the medical oncology service at our hospital. Cardiac tamponade as the initial manifestation of malignancy is rare and such patients have a very limited expectancy, is because at the time of tumor disease diagnosis it is already advanced. There are few reported cases of patients with pericardial tamponade who presented with abdominal pain.
\end{abstract}

Keywords: Cardiac tamponade; Abdominal pain; Tumor manifestation

\section{Introduction}

In $5-15 \%$ of patients with a malignant disease has the pericardium involved, in necropsy series [1]. Metastatic infiltration is most frequent than primary pericardiac tumors.

\footnotetext{
Manuscript accepted for publication May 21, 2013

${ }^{a}$ Emergency Department, Hospital of Navarre, Spain

${ }^{b}$ Public University of Navarre, Health Department, Spain

${ }^{\mathrm{c}}$ Corresponding author: Tomas Belzunegui Otano, Emergency

Department, Hospital of Navarre, Spain.

Email: tomas.belzunegui@unavarra.es
}

doi: http://dx.doi.org/10.4021/jmc1213w
Most frequent tumors are adenocarcinoma and lymphomas. Neoplastic pericarditis can be presented as acute pericarditis, pericardial effusion, effusiveconstrictive pericarditis or cardiac tamponade. For the majority of patients, a clinical manifestation of neoplastic pericarditis is absent or remains unrecognised during their life. Symptoms rarely develop, and even more rarely produce cardiac tamponade. We present the case of a 56 year old man attended on our emergency room with abdominal pain lasting 3 days. Abdominal pain as the first symptom of cardiac tamponade is rare and rare is also cardiac tamponade being the initial manifestation of malignant advanced disease.

\section{Case Report}

56 year old male asymptomatic until three days ago began with intense pain in epigastric region. Constant pain, not irradiated, without regard to meals without nausea, vomiting, or changes in bowel habits. Refer cold 10 days ago.

The initial examination showed: Blood presure was $105 / 70 \mathrm{mmHg}$, pulse 115 beats $/ \mathrm{min}$, temperature $36.3^{\circ} \mathrm{C}$, respiratory rate 20 breaths/min and oxygen saturation $97 \%$ on room air. The yugular vein was distended; breath and heart sounds were slightly decreased. Right-sided abdominal pain especially pronounced in the epigastric and right iliac fossa, his liver was palpable $2 \mathrm{~cm}$ below the right costal margin. No edema was observed in the lower extremities.

His hematologic and biochemical values showed: $14,200 / \mathrm{mm}^{3}$ white blood cell, with $70.7 \%$ neutrophils, $16.5 \%$ lymphocytes. Normal platelet count and hematocrit. Fibrinogen $547 \mathrm{mg} / \mathrm{dl}$, transaminase elevated AST $172 \mathrm{U} / \mathrm{L}$, ALT $185 \mathrm{U} / \mathrm{L}$, Bilirubin $1.2 \mathrm{mg} / \mathrm{dl}$. His electrocardiograma (EKG) showed sinus tachycardia at 115 beats /min with QRS low voltage (Fig. 1), chest radiograph showed a small left pleural effusion and cardiomegaly Grade I (Fig. 2). His abdominal film was unremarkable.

We ask for a surgery first evaluation. They examined the patient and decided to do an abdominal CT. When he returned from the scanner, the patient began to be worse, pallor and sweating skin, dyspnea at rest, nausea with vomiting. Monitoring and new evaluation: On examination we showed 


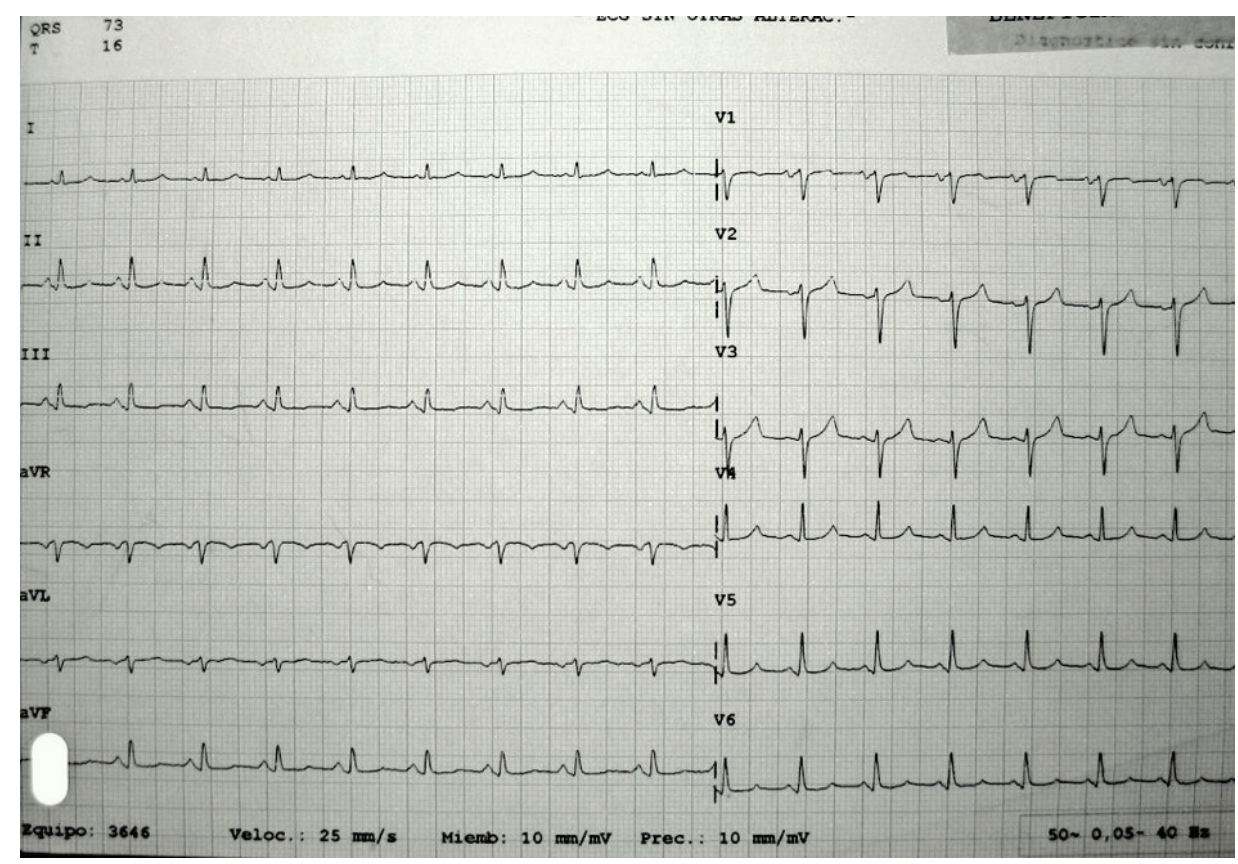

Figure 1. EKG showed Sinus tachycardia, Low-voltage QRS complexes and PR segment depression.

hypotension (blood presure was 90/60 $\mathrm{mmHg}$ ), pulse 100 beats/min, pulsus paradoxus $12 \mathrm{~mm} \mathrm{Hg}$, oxygen saturation $97 \%$ on room air , yugular vein was distended, tachypnea, orthopnea, no change in breath and heart sounds and continued with abdominal pain. In first cut of abdominal CT we showed pericardial effusion (Fig. 3).

With pericardial tamponade suspicion we asked for guard cardiologist evaluation. Confirming the suspect with Echocardiography, large pericardial effusion was observed with right ventricular collapse. Admitted to the coronary care unit, they practiced an emergent pericardiocentesis (subxiphoid acces) and drainage of $940 \mathrm{~mL}$ haemorrhagic fluid. The pacient experiences a rapid improvement in the dyspnea and abdominal pain desappears. After that, the study of pericardial fluid showed malignant cells and elevated tumor

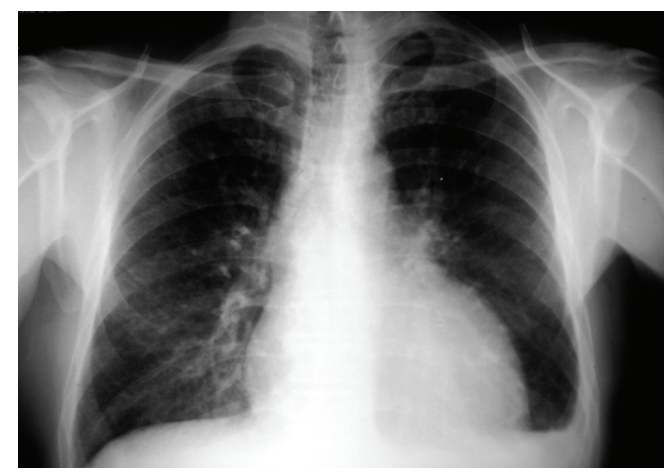

Figure 2. PA chest radiograph showed a small left pleural effusion and cardiomegaly Grade I. markers (CEA $5.861 \mathrm{ug} / \mathrm{L}, \mathrm{Ca} 12516.091 \mathrm{kU} / \mathrm{L})$.

For the study of primary tumor was performed a thoraco-abdominal CT. Showing right hiliar mass, mediastinal lymph nodes, bilateral lung metastases. The patient was diagnosed with stage IV lung carcinoma, initiated the chemotherapy treatment (carboplatin-gemcitabine) by the medical oncology service at our hospital.

\section{Discussion}

Cardiac tamponade as the initial manifestation of malignan-

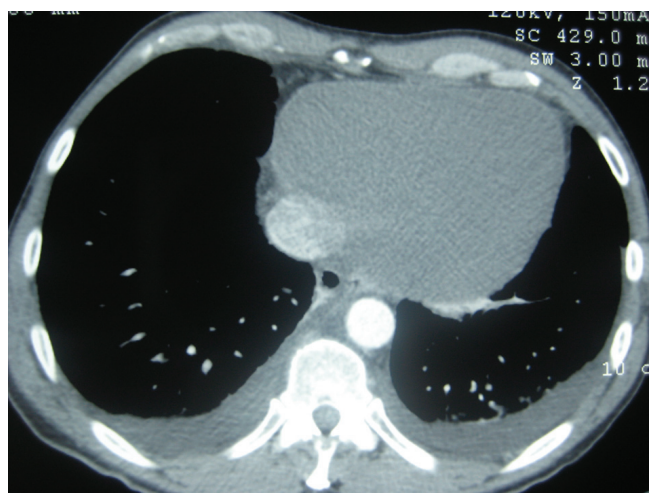

Figure 3. CT scan of the chest shows a large pericardial effusion. Axial unenhanced CT scan shows a homogeneous mass (arrows) that has the same attenuation as water. Contrast-enhanced CT scan shows no enhancement of this mass, a characteristic. 
cy is rare and such patients have a very limited expectancy [2], because at the time of tumor disease diagnosis it is already advanced.

Metastases to the pericardium are much more common than primary tumor, so the search of primary tumor is obligated. No traumatic pericardial tamponade causes are: 40\% Metastases-malignant disease, 15\% Acute idiopathic pericarditis, $10 \%$ Uremia, $10 \%$ Idiopathic bacterial or tuberculous pericarditis , $10 \%$ Cronic idiopathic pericarditis, $5 \%$ Hemorrhage (Anticoagulant), 10\% Others (LES, Postradiation, Myxedema...) [3].

Of that $40 \%$, the most frequent location of primary tumor is in the thorax (77.8\%): lung, breast and lymphoma. $11 \%$ the location is unknown [4].

The clinical suspicion of cardiac tamponade is crucial for the identification and a careful anamnesis and physical examination are the key to a good diagnosis. Cardiac tamponade is always a clinical diagnosis and additional test confirm the suspicion. Beck's triad (hypotension, faint heart sound and yugular venous distention), pulsus paradoxus and Kussmaul's sign is seen with rapid onset tamponade (Obstructive shock). When cardiac tamponade develops slowly, patients are more likely to exhibit dyspnoea, tachypnoea, orthopnoea, tachycardia, hypotension, jugular vein distention, oedema with fovea, ascites, hepatomegaly and abdominal pain [3].

There are few reported cases of patients with pericardial tamponade who presented with abdominal pain [5-8]. Maybe due to hepatic congestion and/or diaphragmatic irritation [3], the hemodynamic and clinical effects of a pericardial effusion depend on fluid volume, rapidity of accumulation and myocardial competence [9].

Additional test are used to confirm the clinical suspicion, etiological diagnosis and assess the severity of the case: Hemathologic, coagulation and biochemical values. Electrocardiogram looks for electric alternance, tachycardia and low voltaje. Chest x-ray looking for enlarged cardiac silhouette, pleural effusion (in rapid onset tamponade we can not show it). Echocardiography documents the presence and magnitude of pericardial effusion. In critically ill patients, when hemodynamic instability precludes echocardiographic diagnosis, emergent pericardiocentesis is diagnostic and therapeutic [10]. The CT can also help the diagnosis and help the further study of etiological origin; is the case of our patient. Citology of pleural and pericardic liquid helps also to ethiologic diagnosis.

\section{Conclusion}

It is therefore an unusual case in which cardiac tamponade is the first manifestation of a malignant tumor and rarer still was the mode of presentation as initial abdominal pain. This case demonstrates once again the great importance of making a good anamnesis and physical examination. The clinical suspicion of cardiac tamponade is crucial for the identification of a patient with malignant cardiac tamponade despite of non-specific symptoms and signs, because chest x-ray, ECG and even echochardiographic findings are not $100 \%$ sensitive or specific, as well. Pericardiocentesis provides diagnosis and offers this group of patients an immediate relief, but trials with various chemoterapeutic agents and radiotherapy, besides, a new surgical procedure will hopefully change the survival for this group of oncologic patients

\section{References}

1. Mukai K, Shinkai T, Tominaga K, Shimosato Y. The incidence of secondary tumors of the heart and pericardium: a 10-year study. Jpn J Clin Oncol. 1988;18(3):195-201.

2. Fraser RS, Viloria JB, Wang NS. Cardiac tamponade as a presentation of extracardiac malignancy. Cancer. 1980;45(7):1697-1704.

3. Niemann JT. The cardiomyopathies, myocarditis, and pericardial disease. In: Tintinalli JE, Kelen GD, Sapsczynski JS, eds. Emergency medicine: A comprehensive study guide. 6th ed. New York: McGraw-Hill; 2004:378386.

4. Eroles Vega G, Rondon Fernandez P, Ferreiro Lopez D, Romero Perez C, Salcedo Perez S, Lacambra Calvet $\mathrm{C}$, Solis Villa J. [Tumor-associated significant pericardiac effusions: analysis of 18 cases]. Rev Clin Esp. 2004;204(5):260-263.

5. Bellamy MC, Natarajan V, Lenz RJ. An unusual presentation of cardiac tamponade. Anaesthesia. 1990;45(2):135-136.

6. Schrager RE, Phillips GR, 3rd, Kauder DR, Schwab CW. Traumatic pericardial tamponade presenting as mesenteric ischaemia. Injury. 1994;25(3):199.

7. Strimlan CV, Turbiner EH. Pleuropericardial effusions associated with chest and abdominal pain. Chest. 1982;81(4):493-494.

8. Muniz AE. Pericardial tamponade presenting as abdominal pain in a patient with systemic lupus erythematosis. CJEM. 2000;2(2):117-120.

9. Reddy PS, Curtiss EI. Cardiac tamponade. Cardiol Clin. 1990;8(4):627-637.

10. Kelly TA. Cardiac tamponade in systemic lupus erythematosus: an unusual initial manifestation. South Med J. 1987;80(4):514-516. 